\title{
Device for Testing the Fatigue Life of Composite Structural Elements of Vehicles
}

\author{
Władysław Papacz ${ }^{1 *}$, Janusz Walkowiak', Peter Frankovský \\ 'University of Zielona Góra, Faculty of Mechanical Engineering, Prof. Z. Szafrana 4, 65-516 Zielona Góra, Poland \\ ${ }^{2}$ Technical university of Košice, Faculty of Mechanical Engineering, Letná 9, 04200 Košice, Slovakia
}

\section{BIOGRAPHICAL NOTES}

Władysław Papacz, Ing. PhD., (1961) Since 2001 he has been working at the Faculty of Mechanical Engineering, University of Zielona Góra as an assistant professor. He graduated from the faculty of construction and operation of machines. His scientific and research work focused in the field of optimization and development of polymer composites structures. Member of Central European Composite Claster and Polish Scientific Society of Motorization.

Janusz Walkowiak, PhD, Since 2002 he has been working at the Department of Mechanical Engineering at University of Zielona Gora as an assistant professor. He graduated from the faculty of the construction and operation of machines. His scientific work and research focuses in the field of processing of plastics and polymer composites.

Peter Frankovský, Ing. PhD. He is a senior assistant on Department of Applied Mechanics and Mechatronics. In 2010 he received PhD. in the field of applied mechanics. He works on scientific and research projects on the department and publishes the results in journals and conference proceedings at Slovakia and abroad. He made remarkable work on the building of laboratories and on publication of monographs and university textbook at the department.

\section{KEYWORDS}

Fatigue tests; Polymer composite, Motor vehicle.

\section{ABSTRACT}

The paper presents the device of the fatigue tests in the complex load states. The measuring system can measure and record the temperature of the elements tested, the number of fatigue cycles, twisting and bending angles of the sample and its load. The device is used to test the fatigue strength of elements of the carrying structures of the vehicles and machines cophasally loaded with the bending and twisting moments.

\section{INTRODUCTION}

Each load-carrying structure put on the dynamic load is subject to the fatigue effect. This is involved with the generation and development of fatigue cracks which lead to weakening and often destruction of the structure. The effect of fatigue is very common in the light, strained structures such as the load-carrying structures of automotive vehicles. Contemporary trends to reduce the energy consumption in vehicles while ensuring the high levels of exploitation parameters result in the increasing use of polymer composites for construction of the load-carrying structure. This forces the search for solution to the problems associated with determining the fatigue life of 
structures made from these materials.

Typical fatigue calculations are performed based on the data of element loads and allowable stresses (the Wohler curve for the harmonic loads). Due to the stochastic nature of the vehicle loads the direct use of such runs to assess the durability of the structure is not possible. So the method of loads reduction for the stochastic runs is used and the Wohler curve is applied. Assuming a suitable hypothesis of fatigue damage culmination the fatigue life of the element can be defined. The accuracy of these calculations depend on the accuracy of the adoption of the correct hypothesis of damage culmination, accuracy of determination of the substitute spectrum of loads and fatigue characteristics of the material. Particularly troublesome is to determine the fatigue strength, the limiting number of fatigue cycles and the Wohler curve exponent. Determination of these parameters, using laboratory testing of the samples of polymer composites, does not produce satisfactory results. For these reasons, the fatigue tests are increasingly being conducted on the real structures or large elements thereof. Such tests should take into account the impact of the type of load on the fatigue life of the designed element. Elements of the vehicle body are mostly bent in the vertical and horizontal planes and twisted. The local load of vehicle subassemblies has less influence on the fatigue life. This paper presents a research stand with the measuring system allowing to conduct the testing of fatigue of elements of machines and vehicles in complex state of loads.

\section{Construction and Operation of the Fatigue Device}

It was assumed that the device should enable the fatigue testing of samples and real elements in a complex state of loads. This state is most common in elements of the carrying structures of automotive vehicles. After review of fatigue testing machines $[1,3]$ the torsion-bending test resonance device with semicircular handles has been chosen. The device allows burdening of a sample with the bending and twisting moments acting cophasally. This case is considered to be the most unfavorable load of the carrying elements of vehicle. Schemes of similar devices have been presented in this paper [2]. The device was built to test the vehicle carrying elements made of polymer composites. In or- der to force the loads, the inertial vibrator driven by three-phase AC motor controlled by a frequency converter was chosen. The maximum length of the element tested is $550 \mathrm{~mm}$, the frequency of forced loads is up to $50 \mathrm{~Hz}$. The angle of deflection of inertial disks is approximately.

The overall construction of the device is shown in Fig. 1. The element tested is fixed in holders mounted on guides of the semicircular forcing elements. The guides are rigidly connected with the inertial disks. On the periphery of the disks the sectional weights are mounted. The inertial vibrator driven by an electric motor is attached to the active disk. The horizontal component of the centrifugal force of the rotating vibrator masses $(4$, fig. 1 ) induces the angular vibration of the active disk. As a result of these vibration the element tested is burden.

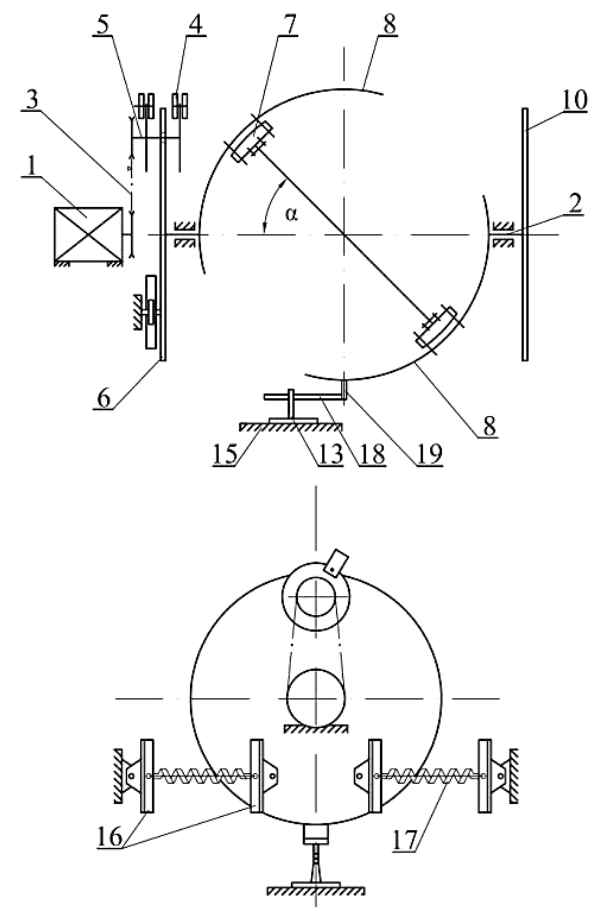

Fig. 1: Scheme of the fatigue device; 1- motor, 2 - main shaft, 3v-belt, 4 and 5 - vibrator weights, 6 i 10 - inertial disks, 7- handles, 8 -semicircular guides.

The use of the semicircular guides allows for optional establishing of the ratio of the bending to the torsion moments depending on the angle of the sample attachment with respect to the horizontal axis of the device $\left(0^{\circ} \leq \alpha \leq 90^{\circ}\right)$. The distribution of moments loading the test sample is 
show in Fig. 2.

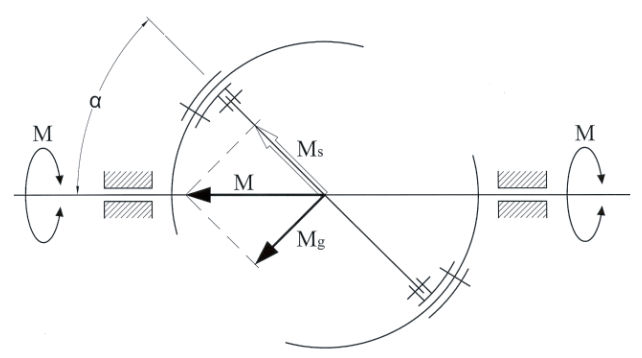

Fig. 2: Distribution of the external moment depending on the angle of the sample setting.

In cases where the bending rigidity and torsion rigidity are equal, which is extremely rare, the moments distribution is as follows:

$$
\begin{aligned}
& M_{g}=M \sin \alpha \\
& M_{s}=M \cos \alpha \\
& \text { If } k_{g} \neq k_{s} \text { then [2]: } \\
& M_{g}=\frac{M}{k_{1}} k_{z} \sin \alpha \\
& M_{s}=\frac{M}{k_{1}} k_{s} \cos \alpha \\
& k_{1}=k_{z} \sin ^{2} \alpha+k_{s} \cos ^{2} \alpha \\
& k_{z}=\frac{E}{\frac{l}{I_{p}}+\frac{2 l_{k}}{I_{k}}} \\
& k_{s}=\frac{G I_{n}}{l}
\end{aligned}
$$

where: $l$ - measuring length of the sample, $l_{k}$ length of the sample mounting terminals, $I_{n}-$ mo- $^{-}$ ment of inertia of the torsion bounded, $I_{p}$-moment of inertia of the measured part of the sample with respect to axis " $z$ ", $I_{k}$-moment of inertia of the sample terminals with respect to axis " $z$ ".

The external moment $M$ can be calculated from the relationship:

$$
M=k \cdot\left(\varphi_{1}-\varphi_{2}\right)
$$

The angle of torsion $\varphi$ can be calculated by solving the system of equations:

$$
\left\{\begin{array}{l}
I_{1} \frac{d^{2} \varphi_{1}}{d t^{2}}+c_{1} \frac{d \varphi_{1}}{d t}+\left(k_{3}+k_{1}\right) \varphi_{1}-k_{1} \varphi_{2}=M_{(t)} \\
I_{2} \frac{d^{2} \varphi_{2}}{d t}+c_{2} \frac{d \varphi_{2}}{d t}-k_{1} \varphi_{1}+\left(k_{1}+k_{2}\right) \varphi_{2}=0
\end{array}\right.
$$

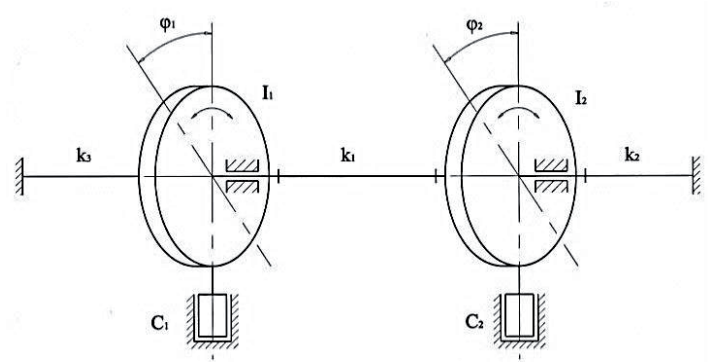

Fig. 3: Dynamic model of the device.

Assuming a solution in the form:

$\varphi_{1}=a_{1} \sin \left(\omega t+\varphi_{01}\right), \varphi_{2}=a_{2} \sin \left(\omega t+\varphi_{02}\right), \varphi=\varphi_{1}-\varphi_{2}$

$\varphi=A_{3} \sin \left(\omega t+\varphi_{0}\right), A_{3}=\sqrt{c_{1}+c_{2}}, \operatorname{tg} \varphi_{0}=\frac{c_{1}}{c_{2}}$

$c_{1}=a_{1} \sin \varphi_{01}-a_{2} \sin \varphi_{02}, c_{2}=a_{1} \cos \varphi_{01}-a_{2} \cos \varphi_{02}$ (11)

$a_{1}=\sqrt{A_{1}^{2}+B_{1}^{2}}, a_{2}=\sqrt{A_{2}^{2}+B_{2}^{2}}, \operatorname{tg} \varphi_{01}=\frac{B_{1}}{A_{1}}, \operatorname{tg} \varphi_{02}=\frac{B_{2}}{A_{2}}$

$A_{1}=\frac{M_{0}\left[A\left(k_{1}+k_{2}-\omega^{2} I_{2}\right)+B \omega c_{2}\right]}{A^{2}+B^{2}}, A_{2}=\frac{M_{0} B k_{1}}{A^{2}+B^{2}}$

$B_{1}=\frac{M_{0}\left[A \omega c_{2}-\left(k_{1}+k_{2}-\omega^{2} I_{2}\right)\right]}{A^{2}+B^{2}}, B_{2}=\frac{-M_{0} B k_{1}}{A^{2}+B^{2}}$

$A=\left[\left(k_{3}+k_{1}-\omega^{2} I_{1}\right)\left(k_{1}+k_{2}-\omega^{2} I_{2}\right)-k_{1}^{2}-\omega^{2} c_{1} c_{2}\right](15)$

$B=\left[c_{1}\left(k_{1}+k_{2}-\omega^{2} I_{2}\right)+c_{2}\left(k_{3}+k_{1}-\omega^{2} I_{1}\right)\right]$

The moment acting on the inertial disk can be calculated from the relationship:

$$
M_{(t)}=M_{0} \cos v t
$$

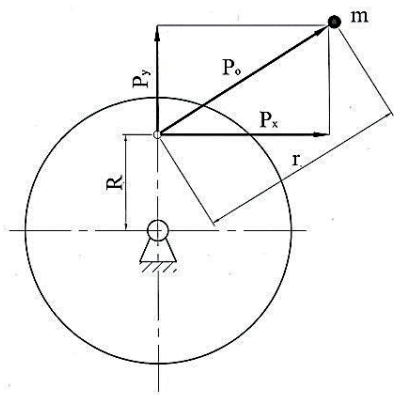

Fig. 4: Centrifugal force on the vibrator; $m$-mass of the weight, $P_{0}$ - centrifugal force, $v$ - frequency of the exciting force. 
While the centrifugal force and the moment in the oscillator are described by the equations:

$$
P_{0}=m r^{2} v^{2} \text { and moment } M_{0}=P_{0} \cdot R
$$

The frequency of free vibration, assuming the same bearings of the disks and identical inertial disks, can be calculated from the relationship:

$\omega_{1 / 2}=\frac{r_{1}+r_{2}}{2 I} \pm \sqrt{\frac{1}{4}\left(\frac{r_{1}+r_{2}}{I}\right)^{2}-\frac{r_{1} r_{2}-k_{1}^{2}}{I^{2}}}$

where:

$$
r_{1}=-I h^{2}+k_{3}+k_{1}, r_{2}=-I h^{2}+k_{1}+k_{2}
$$

\section{The measuring system}

The measuring system can measure and record the temperature of the element tested, the number of the fatigue cycles, the twisting and bending angles of the sample and its load. For registration of the measurements signals the specifically developed measuring system was used. The schematic of the measurement system is shown in Fig. 5.

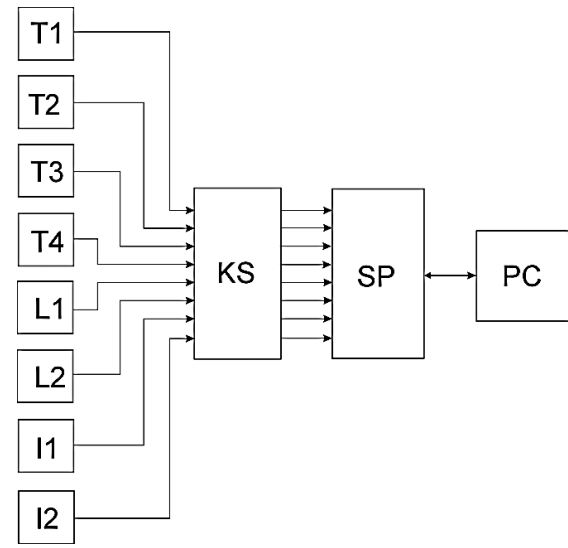

Fig. 5: Microprocessor based measuring system for recording signals during fatigue testing: $P C$ - a control computer, SP - a microprocessor recorder, KS - a signal converter,T1-T4 - silicon sensors of temperature, L1-L2 - potentiometric sensors measuring the angle of deflection, 11 -12 photo-optical sensors to control the cycles of deflection.

The main elements of the measuring system are: PC - a control computer, a microprocessor - based recorder (SP) and a signal converter (KS). The measuring system enables simultaneous, multi-channel measurement and recording of the following values:
- temperatures of the four semiconductor temperature sensors,

- angular deflection from two potentiometric transducers of displacement,

number of deflection cycles from two photo-optical sensors.

The presented configuration of the measurement signals in the first turn allows to determine the self-excited temperature adopted as a criterion of the fatigue destruction. On the surface of the sample tested three silicon sensors were placed. The forth sensor, as a compensation one, is used to measure the ambient temperature. Sensors with a special construction of the signal converter enable the temperature measurement with resolution of $0,01^{\circ} \mathrm{C}$. The adopted solution provides measurement and recording of even minimal changes of temperature on the surface of the sample tested. The idea of measuring the twist angle a of the sample is shown in Fig. 6.

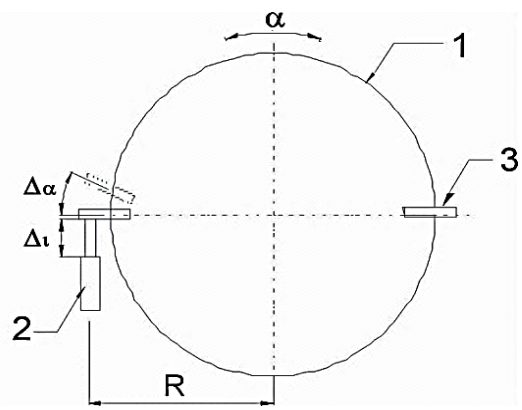

Fig. 6: Measurement of the angle of torsion of the sample: 1 -movable disk of the forcing unit, 2 -potentiometric sensor of displacement, 3 -pillar.

The signal obtained directly at the output of the potentiometric converter of displacement (2) is the signal that is information on linear displacement $\Delta 1$ of the mandrel (slider) of the potentiometer forced by the angular movement $\Delta a$ of the support coupled with that mandrel in "a floating" system (3). In order to measure and record the angle of torsion a of the sample, the appointment and maintaining of the processing characteristics were done at the beginning which are described with the relationship:

$$
\Delta \alpha=f(\Delta U)
$$

The accepted concept of construction of the measuring system of the open configuration enables, in a simple manner, connecting of additional 
measuring transducers, eg. strain gauges to measure the deformation in the attachment points of the element tested. In Fig. 7 the arrangement of transducers are schematically shown.

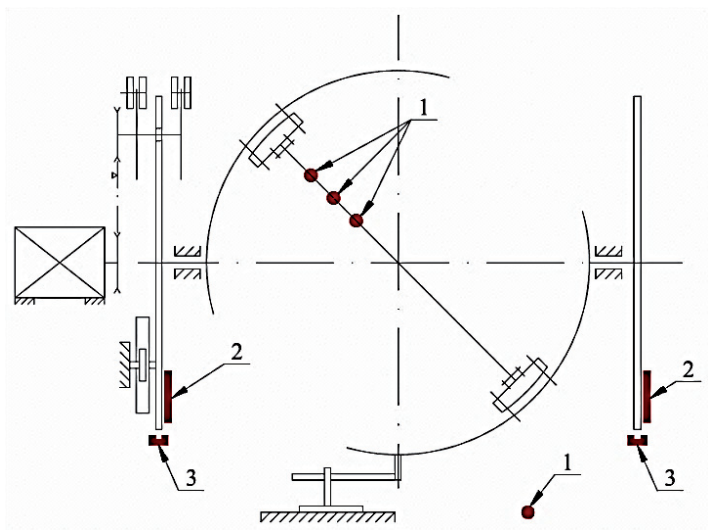

Fig. 7: Placement of the measuring sensors: 1 - temperature sensors, 2 - sensors of the angular displacements of disks, 3 - sensors of the fatigue cycles counter.

\section{Research Possibilities}

The fatigue device inductor is powered by a three-phase AC motor through a belt transmission. The control of rotational speed is achieved by the frequency inverter type RN82 LUMEL. The forced loads frequency can be adjusted by the change of the motor rotations, while the moment of loading of the element tested can be regulated by changing the mass of the weights attached to the vibrator disks. Reactive guide in its lower part is connected to the base of the device with an elastic connector. The rigidity of the connector can be adjusted. Similarly, the active disk is connected to the base by coil springs and pillars. Using the changes in the stiffness of connectors of both disks and their moments of inertia the dynamic model of the device can be changed from the dual mass system with one natural frequency to the system with two frequencies of natural vibrations. Below, in the Fig. 8, 9 and 10 the possibilities of loading the element tested are shown. Depending on the angle of inclination of the sample, various possibilities of its load are achieved. From loading with torque moment only through a complex state of loading (bending and twisting moments) to loading solely with the twisting moments). The device allows therefore for conducting the high-cycle fatigue tests of samples and construction elements.

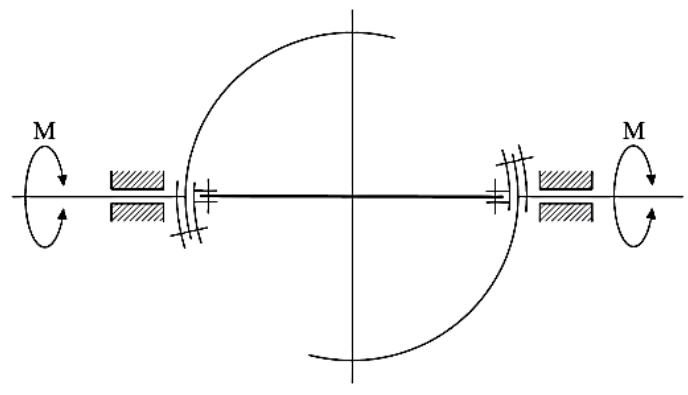

Fig. 8: Loading with the twisting moment MS.

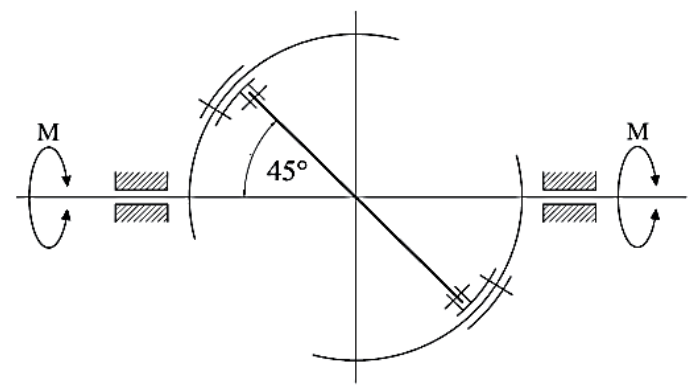

Fig. 9: Cophasal loading with the bending $M_{g}$ and twisting $M_{s}$ moments.

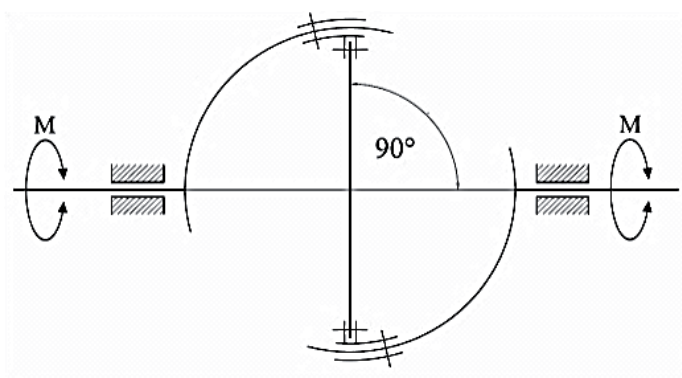

Fig. 10: Loading with the bending moment $M_{g}$.

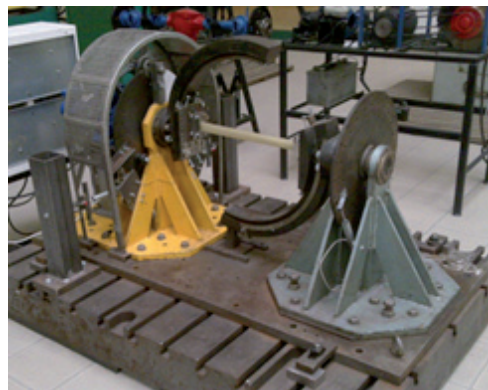

Fig. 11: View of the research stand with a composite sample.

\section{Conclusions}

The paper presents the stand of the fatigue tests in the complex load states. The measuring system can measure and record the temperature of the elements tested, the number of fatigue cycles, twist- 
ing and bending angles of the sample and its load. The device is used to test the fatigue strength of elements of the carrying structures of the vehicles and machines cophasally loaded with the bending and twisting moments. It is possible to equip the device with stereoscopic microscope with a camera recording with high resolution (eg Moticam 1000) for observation and measurement of fatigue crack propagation.

\section{References}

[1] Kocańda St., Szala J. Basis of calculation of fatigue, (in polish) PWN 1997,

[2] Katarzyński, S., Kocańda S., Zakrzewski M., Research Mechanical Properties of Metals, (in Polish) WNT, W-wa 1967,

[3] Paluch, Z:: The effect of twisting on the fatigue life of a constrained thin-walled frame vehicles. IKiEMPW, (in Polish),
Wrocław (1984),

[4] Robak, G., Gasiak, G.,: Test kit to measure the development of fatigue cracks at loads of complex,(in polish) PM 2/2008

[5] http://www.zwick.de/pl/produkty/zmeczeniowe-maszynywytrzymalosciowe/pulsatory-rezonansowe-od-5-do-550kn.html(25.01.2013).

[6] Trebuňa, F, Frankovský, P, Bocko J, Pastor M.: New posibilities of using PhotoStress method. Acta Mechanica Slovaca, 15(4) 2011, pp. 44-51.

[7] Kuczyński Z:: Mechanics of motor vehicles (in Polish), WSiP, Warszawa (1975).

[8] Gasiak, G.: Durability of construction materials with cyclic loads involving the average load (in Polish), Opole University of Technology, Opole 2002.

[8] Gasiak, G., Grzelak, J., Robak, G.: Modeling the sustainability of the development of fatigue cracks in bending and torsion (in Polish), Mechanics nr 304, Opole 2005.

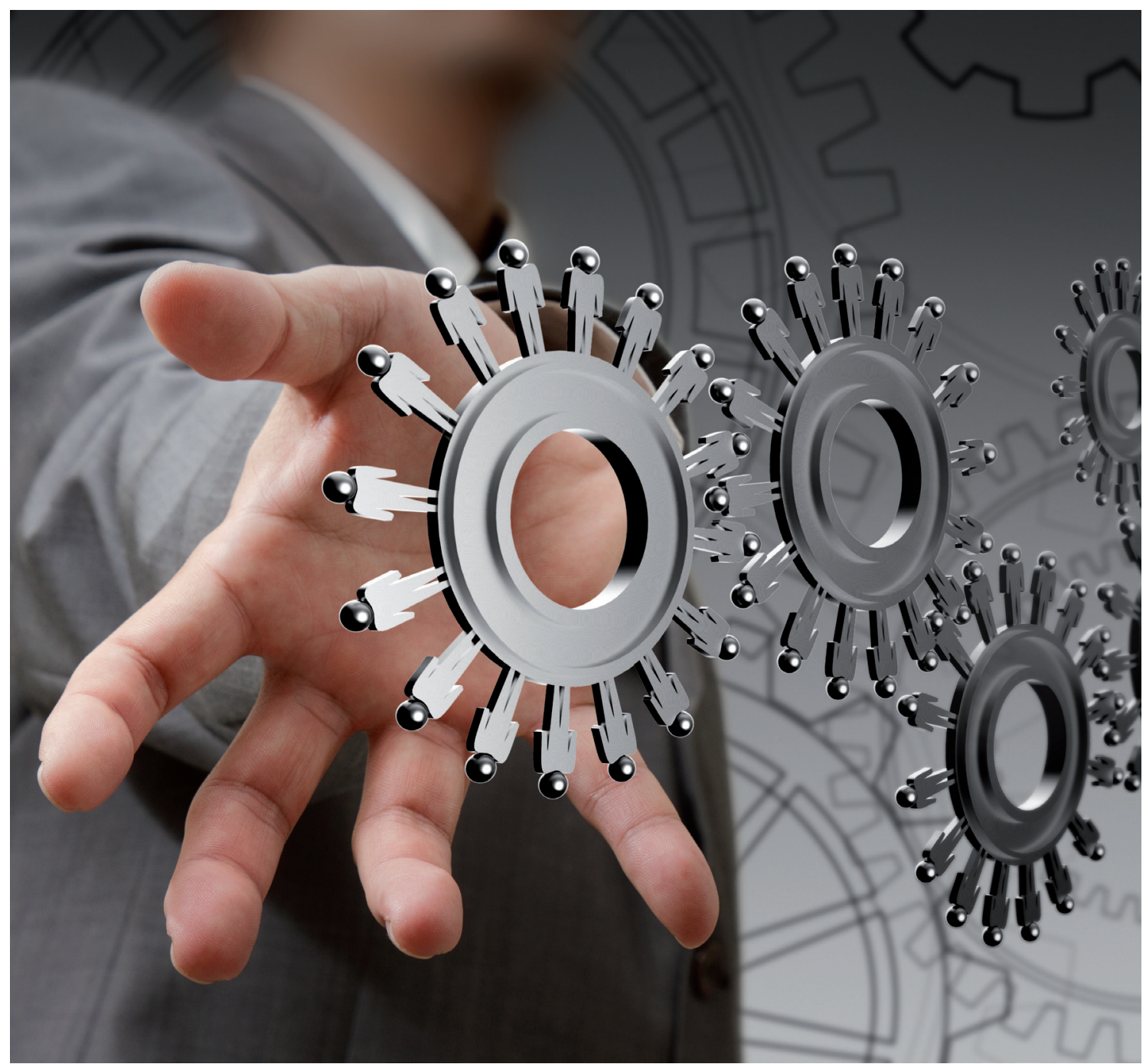

